\title{
Quantum vacuum energy, gravity manipulation and the force generated by the interaction between high-potential electric fields and zero-point-field
}

\author{
Luigi Maxmilian Caligiuri ${ }^{1,2,}$, Takaaki Musha ${ }^{1,3}$ \\ ${ }^{1}$ Foundation of Physics Research Center, FoPRC, via Resistenza 87053 Celico (CS), Italy \\ ${ }^{2}$ University of Calabria, via P. Bucci 87036 Arcavacata Di Rende (CS), Italy \\ ${ }^{3}$ Advanced Science-Technology Research Organization, Yokohama, Japan
}

Email address:

caligiuri@foprc.org. (L. M. Caligiuri), takaaki.mushya@gmail.com (T. Musha)

\section{To cite this article:}

Luigi Maxmilian Caligiuri, Takaaki Musha. Quantum Vacuum Energy, Gravity Manipulation and the Force Generated by the Interaction between High-Potential Electric Fields and Zero-Point-Field. International Journal of Astrophysics and Space Science. Special Issue: Quantum Vacuum, Fundamental Arena of the Universe: Models, Applications and Perspectives. Vol. 2, No. 6-1, 2014, pp. 1-9. doi: 10.11648/j.ijass.s.2014020601.11

\begin{abstract}
The idea of manipulating and using the energy associated to electrodynamic quantum vacuum, also known as Zero Point Energy (ZPE), for technological applications as, for example, interstellar space propulsion, represents one of the most challenging question both in theoretical and applied physics. During the past years B.Haish, A.Rueda and H.E.Puthoff proposed a model according to which inertia could be considered as the electromagnetic reaction force to interaction between a body and quantum vacuum zero point field (ZPF), opening interesting perspectives about manipulating inertia by electromagnetic fields. Nevertheless this theory, although interesting from both a theoretical and applicative point of view, is for from being complete and presents some questionable points. More recent results have suggested a novel model of quantum vacuum, ruled by "Planck metric" and characterized by an energy density field, able to give a novel interpretation of mass and gravity in terms of variation of such energy density. In this paper we'll propose an extension of this model allowing the theoretical possibility of inertia and gravity strength manipulation, as well as a more fundamental theoretical explanation of some assumptions of the Haish, Rueda and Puthoff model. In particular, it will be shown that not only inertia but gravitational "constant" as well can be expressed as functions of quantum vacuum energy density, analyzing their relationships with the electromagnetic field, described by vector potential. Finally we will discuss the possibility of space propulsion system by considering the interaction between the zero-point field of the quantum vacuum and the high potential electric field generated in an asymmetrical capacitor, showing the resulting force is driven by quantum vacuum energy density.
\end{abstract}

Keywords: Zero Point Field, Quantum Vacuum Energy Density, Gravity Manipulation, HRP Model, Space Propulsion, Asymmetrical Capacitor, Electrogravity

\section{Introduction}

In modern physics the "vacuum" cannot be considered, due to Heisenberg uncertainty principle, as a void but as physical entity manifesting a complex and fundamental background activity in which, even in absence of matter, processes like virtual particle pair creation - annihilation and electromagnetic fields fluctuations, known as zero point fluctuations (ZPF) continuously occur (see Fig.1). The physical reality of ZPF, also known as Quantum Vacuum (QV), has been proved to give measurable effects for the first time by Casimir's experiment in 1948 [1] and next confirmed by
Lamoreux [2], Milonni et al. [3]. From a more theoretical point of view, Russian physicist Andrei Sakharov, in searching for deriving Einstein's phenomenological equations for general relativity from more fundamental set of assumptions, came to the conclusion that general relativistic phenomena could be seen as induced effects brought about by changes in the quantum-fluctuation energy of the vacuum due to the presence of matter [4].

Based on the Sakharov's hypothesis, Puthoff [5] explored a conceptually simple, classical model according to which gravitation could be not a fundamental interaction at all, but rather an induced effect due to changes in the quantum-fluctuation energy of the vacuum when matter is 
present.

Within this model Puthoff proposed the hypothesis that ordinary matter is ultimately made of sub-elementary constitutive primary charged entities (the so called "partons") bound in the manner of traditional elementary oscillators and able to dynamically interact with fluctuating ZPF, according to a sort of resonance mechanism. In more details, in his model, a material body should be considered, with respect the electromagnetic interaction, as a resonant cavity in which a suitable set of ZPF modes oscillates. On this basis, Hairsh, Rueda and Puthoff (HRP) proposed a model in which the inertial mass associated to a body, characterized by a proper volume $V_{0}$ (the "electromagnetic equivalent volume"), is given by [6]:

$$
m_{i}=V_{0} / c^{2} \int \eta(\omega) \rho(\omega) d \omega
$$

in which $\omega$ is the angular frequency of ZPF mode, $\rho(\omega)$ is the spectral energy density of quantum vacuum ZPF fluctuations and $\eta(\omega)$ is a function that would quantify the fraction of ZPF energy density that electromagnetically interacts with the particles contained in the "useful volume" $V_{0}$ or, in other words the "efficiency" of interaction [6]. In this way the apparent inertial mass of a given object would originate by the interaction, during the accelerated motion of the body, between the ZPF energy density fraction enclosed in the object (given by $\eta(\omega)$ ) and the partons contained in the volume $V_{0}$.

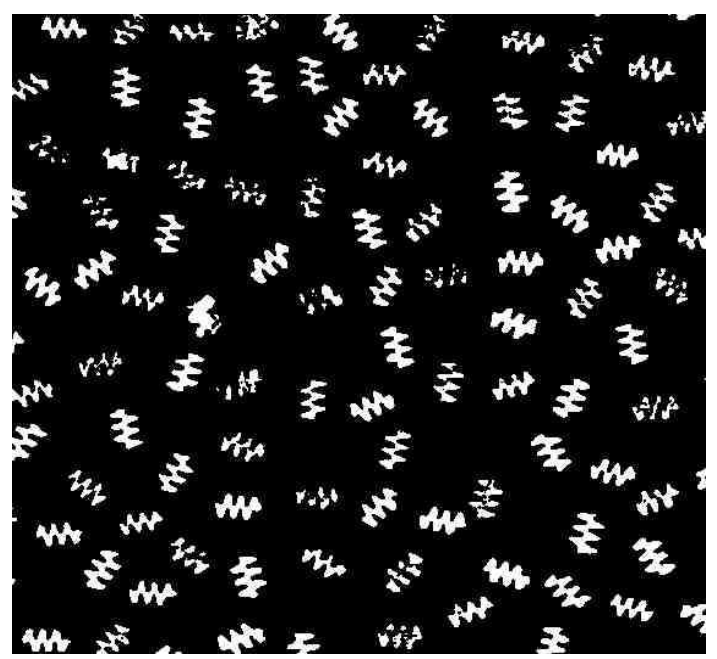

Figure 1. A possible representation of ZPF energy distribution in quantum vacuum.

In HPR model the inertial mass so defined also coincides with the rest mass of Einstein's Special Theory of Relativity so that the rest energy $E=m_{i} c^{2}$ would represent the quantity of ZPF energy electromagnetically interacting with charged elementary partons, co - moving with the accelerated body as viewed by an inertial observer. By means of relativistic reasoning, involving the demonstration of the equivalence between the force in a reference frame fixed above a gravitational field and the inertia force associated to (1) in an accelerated frame, HRP deduced the weak equivalence principle of relativity and then assumed [6]

$$
m_{g}=m_{i}=V / c^{2} \int \eta(\omega) \rho(\omega) d \omega
$$

where $m_{g}$ is the gravitational mass of the considered body, and interpreted gravity as a form of long-range van der Waals force associated with the "Zitterbewegung" of elementary particles in response to ZPF fluctuations of QV.

This interpretation of inertial and gravitational masses as the result of the interaction between matter and ZPF suggests the possibility to modify the inertia of a body and the gravitational force acting on it by altering ZPF through electromagnetic interaction. This idea has very deep implications both theoretical and technological as, for example, the possibility of inertia manipulation for space propulsion [7].

Although the HRP model is very interesting and without doubt able to give fundamental insights into the QV dynamics and the origin of inertia and gravitation, it faces with some open questions, briefly cited in the following discussion, whose more detailed analysis will be discussed in a forthcoming paper [8]. The first one is related to the type of QV energy density described by the function $\rho(\omega)$ that is electromagnetic only.

Nevertheless, according to the Standard Model (SM), the total vacuum energy density includes at least three contributions: the electromagnetic fluctuations characterizing the zero-point field (ZPF), the fluctuations characterizing the quantum chromo-dynamic level of sub-nuclear physics and the fluctuations linked with the so-called Higgs field. Moreover, one can speculate that there are also contributions from possible existing sources outside the SM (for instance, grand unification theories, string theories, etc.). In this sense the HRP model could result incomplete since it doesn't consider the contributions from these other possible sources of QV energy density (conversely, the SM is far from representing a complete and definitive picture of physical reality, being it affected by worrying defects and deficiencies as, in particular, its inability to describe the fundamental force of gravity and the presence of so much matter in comparison to anti - matter). A second question is related to the "efficiency" function $\eta(\omega)$, phenomenologically implying some type of "resonant" interaction between the partons included in the body and the ZPF fluctuations contained in the volume cavity, introduced in the model as a free parameter and whose physical origin, from a dynamical point of view, is not explained in the model (as well as that of the resonant cavity of volume $V_{0}$ associated to any given material body). The above cited questions as well as other questionable aspects of the HRP model could be overcame by means of its reinterpretation within a more general model of $\mathrm{QV}$ being in progress [8].

Recently, a new model of $\mathrm{QV}$, consisting of a granular structure composed by energetic packages having the size of Planck's volume $V_{P}=l_{P}^{3}$ (where $l_{P}$ is the Planck's length) and characterized by a dynamic energy density $\rho(\vec{r}, t)$ in which rest and relativistic mass of a body arise from the local variation of QV energy density, has been proposed by one of 
the authors $[9,10]$. Within this model, gravity can be interpreted as dynamically originated by QV energy density gradients $\Delta \rho(\vec{r}, t)$ due to the presence, in the $3 \mathrm{D}$ physical space, of massive bodies and particles $[9,11]$.

In this paper a reformulation and extension of this model, whose more detailed treatment is discussed in a dedicated paper [8], in which the gravitational constant $G$ is expressed as a function of QV energy density, is presented. According to this interpretation, not only the inertial component of gravitation (related to gravitational masses) but also gravity strength itself (as described by $G$ ) depends upon QV energy density. In this way, by acting on this energy density, it is in principle possible to modify both gravitational inertia and gravity strength so offering the possibility to "manipulate" gravitational force according to a wide range of possibilities, in particular by means of electromagnetic interaction.

A possible link between HRP model of gravitation and the one here proposed is also presented.

Finally we will discuss the very interesting possibility of space propulsion system by considering the interactions between the zero-point field of $\mathrm{QV}$ and the high potential electric field generated in an asymmetrical capacitor, showing the resulting force is driven by QV energy density and considering the possibility of propulsion system based on the modification of its inertial mass, or gravitational strength, by acting upon the zero-point fluctuations of QV.

\section{Gravitational Force as a Consequence of Quantum Vacuum ZPF Mass-Density Equivalent}

In some previous works $[9,10,11]$ a novel model of $\mathrm{QV}$, ruled by the dynamics of its own energy density, has been proposed. Within this model, physical space is composed by Planck's volumes and the presence of a material object in a given area of 3D physical space diminishes the QV energy density inside a material object or particle. Under the assumption of a uniform QV energy distribution within the Universe, the energy density conservation leads to

$$
\rho_{Q V}+\rho_{e m}+\rho_{M}=\rho_{0}
$$

where $\rho_{Q V}$ is the QV energy density, $\rho_{e m}$ the non fluctuating electromagnetic energy density, $\rho_{M}$ is the relativistic energy density and $\rho_{0}$ is a universal constant value.

From (3), the QV energy density $\rho_{Q V}$ has its maximum value $\rho_{Q V, \max }$ in the absence of matter and radiating non fluctuating electromagnetic fields. In order to assume that Einstein's General Theory of Relativity to remain valid up the Planck scale, we must have

$$
\rho_{Q V, \max }=m_{P} c^{2} / l_{P}^{3}
$$

where $m_{P}$ is the Planck mass and $l_{P}$ the Planck length, whose currently accepted values respectively are $2.177 \times 10^{-8} \mathrm{~kg}$ and $1.616 \times 10^{-35} \mathrm{~m}$ when $G=6.67384 \times 10^{-11} \mathrm{~m}^{3} \cdot \mathrm{kg}^{-1} \cdot \mathrm{s}^{-2}$

The value of $\rho_{Q V} \approx 10^{113} \mathrm{~J} \cdot \mathrm{m}^{-3}$ so obtained by (4), can be considered as the maximum possible value $\rho_{Q V, \max }$ of $\mathrm{QV}$ energy density, since it would represent, within the currently accepted picture, the maximum energy density can exist "without being unstable to collapsing space-time fluctuations" [6] associated to the value $G=6.67384 \times 10^{-11} \mathrm{~m}^{3} \cdot \mathrm{kg}^{-1} \cdot \mathrm{s}^{-2}$ currently assumed.

According to the above results, we can then consider that every particle is made out of electromagnetic energy of QV and so it consists of decreased energy density of an ideal QV. For mass-less particles, the decrease of energy density corresponding to the "creation" of a particle of energy $E=\hbar \omega$ is given by

$$
\rho_{Q V, E}^{\prime}=\left(m_{P} c^{2}-\hbar \omega\right) / l_{P}^{3}
$$

where $\rho_{Q V, E}^{\prime}$ is the QV energy density after the "creation" of the mass-less particle. For a massive particle of rest mass $m$ we have instead

$$
\rho_{Q V, \mathrm{~m}}^{\prime}=\rho_{Q V}-m c^{2} / V
$$

where $V$ is the proper volume of the body and the energy density variation can be considered to be concentrated, for our purpose in this paper, in the center of mass of body or particle.

According to (5) and (6) particles are generated by QV electromagnetic field modes.

From (6) it immediately follows that mass can be expressed as a result of the variation of energy density of an "electromagnetic" QV

$$
m=\left(\rho_{Q V}^{\prime}-\rho_{Q V, m}\right) V / c^{2}
$$

or equivalently

$$
m=\Delta E_{Q V} / c^{2}
$$

having defined $\Delta E_{Q V}=\left(\rho_{Q V}^{\prime}-\rho_{Q V}\right) V$.

Introducing the concept of energy density of $\mathrm{QV}$, the gravitational field existing at a point placed at distance $r$ from the center of a given material object of mass $m$, given by (8) can be written as

$$
\vec{g}=\left(G V / r^{2}\right)\left(\Delta \rho_{Q V, m} / c^{2}\right) \hat{r}
$$

where $\hat{r}$ is the unitary vector indicating direction and orientation of $\vec{r}$.

We now focus on the relationship between the gravitational "constant" $G$ and QV energy density and its consequences upon inertia and gravity [8]. In the proposed model the more fundamental physical entity of the 3D granular space are represented by Planck's units, so it is natural [12] to use them in order to express the value of $G$ as a function of these by noting that, dimensionally

$$
[G]=[L]^{3} \cdot[M]^{-1} \cdot[T]^{-2}
$$


and

$$
\left[\rho_{Q V}\right]=[M] \cdot[L]^{-3}
$$

where we indicate for simplicity with $\rho_{Q V}$, from now on, the so-called Mass - Density - Equivalent (MDE) of QV energy density (equal to $\rho_{Q V} / c^{2}$ where $\rho_{Q V}$ is the originally defined QV energy density function) referring to it simply as QV energy density, so we can write

$$
G=1 /\left(\rho_{Q V} \cdot t_{P}^{2}\right)
$$

where $t_{P}$ is the Planck's time whose value is $t_{P}=5.391 \times 10^{-44} \mathrm{~s}$ when $G=6.67384 \times 10^{-11} \mathrm{~m}^{3} \cdot \mathrm{kg}^{-1} \cdot \mathrm{s}^{-2}$. We can then assume that also $\mathrm{G}$ is a function of $\mathrm{QV}$ energy density and that it defines a fundamental properties of space itself originated from QV

$$
G=l_{P}^{3} /\left(m_{P} c^{2} t_{P}^{2}\right)
$$

Equation (12) can be naturally generalized to the case of a variable $\mathrm{QV}$ energy density by formally assuming

$$
G\left(\rho_{Q V}\right)=1 /\left(\rho_{Q V} t_{P}^{2}\right)
$$

The gravitational field given by (9), due to a mass $m$, can then be generalized as

$$
\vec{g}\left(\rho_{Q V}\right)=\left(1 / \rho_{Q V} \cdot t_{P}^{2}\right) m\left(\rho_{Q V}\right)\left(1 / r^{2}\right) \hat{r}
$$

Equation (15) clearly shows the gravitational field is a property of space that directly derives from the change of the energy density of $\mathrm{QV}$ in the pertinent volume of physical space

According to the above model, the QV energy density inside and around a mass is modified by the presence of the mass itself. We can schematically represent the diminished energy density region associated to a given mass as the light area in Fig. 2.

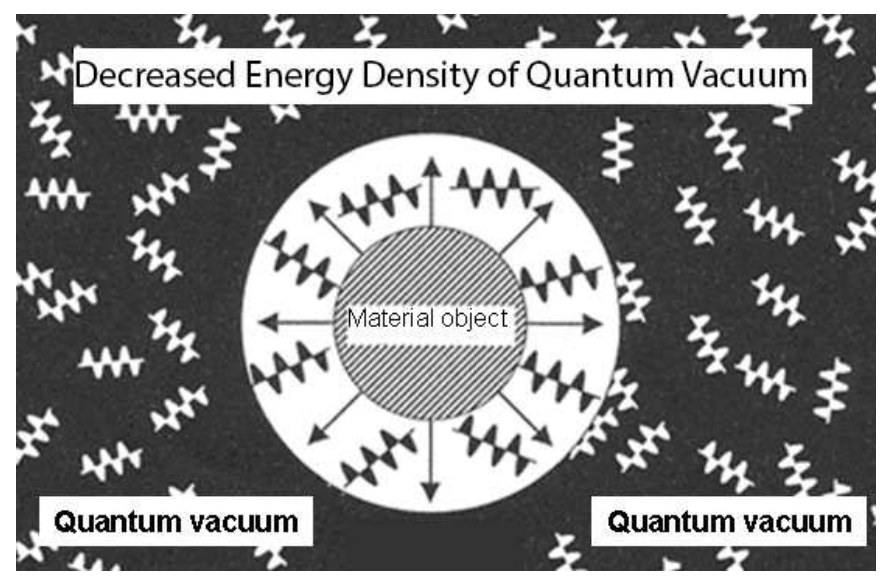

Figure 2. Presence of a given material object diminishes energy density of Quantum Vacuum and this generates inertial mass and gravitational mass.

This mechanism determines a portion of space, around the body, characterized by the presence of a ZPF energy density gradient, whose dependence on distance from the mass determines the dependence of the gravitational potential on the same distance as well. From a dynamical point of view, the area of higher energy density of outer QV "pushes" towards the area of lower energy density due to the presence of the massive body or elementary particle, generating gravity attraction towards the mass, whose strength is proportional to QV energy density gradient $\Delta \rho(\vec{r}, t)$.

This theoretical model also suggests, as already shown [10], a simple explanation of the equivalence between inertial and gravitational mass, both being originated by the same QV energy density decrease for a given massive body or particle (see Fig. 2).

From (15) we see gravity varies when QV energy density does due to two factors:

a) the variation of the value of mass, generating the gravitational field;

b) the modification of the "strength" of gravitational interaction due to the different value of $G$

In particular, if $\rho_{Q v}$ varies so that $\mathrm{G}$ decreases it is possible to simulate an "anti - gravitational" effect. Even in this case, the effect could arise both from gravitational inertia and gravity strength $(G)$ variations .

\section{Gravity Control Through Quantum Vacuum Energy Density Manipulation}

The above discussion shows that gravity could be considered as a function of QV energy density since both $G$ and gravitational mass can be expressed as QV functions. This means that, from a theoretical point of view, gravity manipulation is possible, provided that we are able to modify the local value of $\rho_{Q v}$, for example by altering ZPE.

The first experimental evidence about this concrete possibility came from the experiment conducted by Podkletnov [13] in which a composite bulk YBa2Cu3O7-x superconductor, at a temperature below $70^{\circ} \mathrm{K}$ interacting with a suitable e.m. field, manifested "weak gravitation shielding" in the space below and above the superconductor apparatus.

The same experiment was subsequently reproduced by $\mathrm{Li}$ et al. [14] and analyzed by Modanese [15].

According to Li's conclusions "rotating superconductors in an alternate magnetic field would generate gravity", so suggesting a primary role of electromagnetic energy in modifying the quantum vacuum ZPE and then, for the above considerations, its energy density $\rho_{Q v}$.

From a dynamical point of view, the manipulation of gravity, through ZPE alteration, is based on the possibility to transfer one or more photons from or to QV. In particular, in the gravity shielding experiments involving superconductors and magnetic fields, the reduction of gravity would be obtained by increasing the local value of QV energy density $\rho_{Q v}$, transferring photons from e.m. fields to QV.

This would increase the QV pressure, consequent to the raised photon density of ZPE whose effect would be the lowering of the weights of the bodies placed in the considered space region.

The effect is qualitatively explained very simply within the 
proposed model of QV. In fact, from (3) we have, if we assume $\Delta \rho_{m}=0$ (i.e. no modification of relativistic energy of the bodies)

$$
\Delta \rho_{Q V}=-\Delta \rho_{e m}
$$

namely a decrement of e.m. energy density (photons density) will correspond to an increase of local QV energy density and, by (14), to a gravity weakening.

The same mechanism could be able to qualitatively explain, as already suggested in a previous paper [11], the physical origin of the so called Dark Energy (DE) in the Universe as well as the observed deviation from Newton gravitation law in the rotating galaxies, ascribed to the so called Dark Matter (DM).

The described effect could have enormous implications both theoretical related, for example, to the unification between gravity and electromagnetism (the so called "electrogravity"), and technological related, for instance, to the realization of advanced space propulsion systems (as in the NASA High Temperature Superconductor Research Program).

\section{On a Possible Link between HRP Model and QV Energy Density Dynamics}

As recalled in the introduction, the HRP model intends to explain the physical origin of inertia and gravity as arising from the interaction between the elementary charged components of matter and a suitable fraction of electromagnetic ZPF confined within an "ideal" resonant cavity associate to a given massive body. In doing this it considers the ZPF energy density as obtained by the summation of all electromagnetic plane waves of the ZPF given by

$$
\rho_{Z P F}=\int_{0}^{\omega_{c}} \hbar \omega^{3} / 2 \pi c^{3} d \omega
$$

where $\omega_{\text {is }}$ the angular frequency of ZPF plane wave and $\omega_{c}$ is the so called "high frequency cutoff". This represents the highest frequency component of the spectrum and its presence is firstly motivated by the highly divergent behavior of energy density when $\omega \rightarrow \infty$ that, without an upper cutoff, will determine an infinite value of $\rho_{Z P E}$. In the second place the it prevents the eventual asymptotically high frequency instability of QED itself [6].

For relativistic reasons [6], the high frequency cutoff is assumed to be equal to the Planck frequency

$$
\omega_{c}=\omega_{P}=\left(c^{5} / \hbar G\right)^{1 / 2}
$$

This quantity plays a crucial role in the original version of HRP model since it determines the inertial and gravitational masses associated with the "parton" oscillator

$$
m_{i}=m_{g}=\Gamma \hbar \omega_{c}^{2} / 2 \pi c^{2}
$$

where $\omega_{c}=\omega_{P}$ and $\Gamma$ is Abraham-Lorentz damping constant of the oscillating parton.[16].

If we now consider (18), we see the cutoff frequency is expressed as a function of gravitational "constant" $G$ that is, in turn, a function of QV energy density as previously shown. We can then substitute (14) into (18) to obtain

$$
\omega_{c}=\left(c^{5} t_{P}^{2} \rho_{Q V} / \hbar\right)^{1 / 2}
$$

showing that the cutoff frequency is also a function of QV energy density. It is also interesting to consider the expression of the maximum quantum vacuum ZPF energy density corresponding to (17) when $\omega_{c}=\omega_{P}$, namely

$$
\rho_{Z P F}=2 \pi^{2} c^{7} / \hbar G^{2}
$$

We can now stress that the maximum ZPF energy density calculated by (21) is just, within a factor of $2 \pi^{2}$, the maximum value of QV energy density $\rho_{Q V \text {,max }}$ defined by (4), namely

$$
\rho_{Z P F}=2 \pi^{2} \rho_{Q V, \max }
$$

In fact, starting by the definition of Planck time

$$
t_{P}=\left(\hbar G / c^{5}\right)^{1 / 2}
$$

we can write (squaring (23)) and multiplying side by side by $\rho_{Q V} \neq 0$ )

$$
\rho_{Q V} t_{P}^{2}=\rho_{Q V}\left(\hbar G / c^{5}\right)
$$

but the left right of (24) is just equal to $1 / G$ so we have

$$
1 / G=\rho_{Q V}\left(\hbar G / c^{5}\right)
$$

then

$$
1 / G^{2}=\rho_{Q V} \hbar / c^{5}
$$

Substituting (26) into (21) we obtain the (22) that physically represents, as already said, the maximum energy density the Planck volume can sustain without collapsing if we assume Planck metric to hold. Equation (22) then shows that the quantity $\rho_{Z P F}$ on which HPR model is based and the quantity $\rho_{Q V}$ on which the model proposed in this paper is based have, from a physical standpoint, substantially the same origin.

We can see from the above discussion that both the mass associated to the partons in HRP model and the ZPE energy density calculated with the HRP cutoff can be expressed as a function of QV energy density so suggesting a direct linking between the model here proposed and the HRP one. Such connection will be considered in the following discussion. 


\section{Shift of the Cut - Off Frequency of ZPF Induced by Electromagnetic Fields on Quantum Vacuum}

Recent works imply that ZPF may play an even more significant role as the source of inertia and gravitation of matter [17]. Furthermore, this close link between electromagnetism and inertia suggests that it may be fruitful to investigate to what extent the fundamental physical process of electromagnetic radiation by accelerated charged particles could be interpreted as scattering of ambient ZPF radiation.

The expression for inertial mass derived by HRP involves two free parameters, $\Gamma$ and $\omega_{c}$, the latter assumed by HRP to represent some cutoff frequency dictated either by an actual cutoff of the ZPF spectrum or by a minimum size of an elementary particle $[6,8]$.

If we assume the cutoff frequency to be a resonant frequency $\omega_{0}$, which is specific to a given particle, then the mass of a particle can be converted by external boundary conditions. Haisch, Rueda and Puthoff suggested that if one could somehow modify the vacuum medium then the mass of a particle or object in it would change according to the zero-point field modification.

In a first approximation of the theory, we assume the resonant frequency $\omega_{c}$ can be considered as somehow related to the Compton frequency $\omega_{\text {Comp }}$ of the elementary particle being considered, so we can put

$$
\omega_{c} \approx \omega_{\text {Comp }}=m_{0} c^{2} / \hbar
$$

where $m_{0}$ is the rest mass of the particle.

Under an intense electromagnetic field, it has been theoretically predicted that electron experiences an increase of its rest mass. This effect is also predictable within the QV model based on energy density dynamics discussed at par. 2, since to an increase of $\rho_{e m}$ can correspond, through (3), a decrease of $\rho_{Q V}$ and a corresponding raise to the matter component $\rho_{m}$, namely, by (7), an increase of rest mass of the particle in the considered region of space.

Let $H_{A}$ be the electrodynamic Hamiltonian of the particle under high electromagnetic field, it has the form shown as [3]

$$
H_{A}=\left(e^{2} / 2 m_{0} c^{2}\right)<A^{2}>
$$

where $m_{0}$ is the rest mass of the particle, $e$ is its charge and $\vec{A}$ is the vector potential.

A similar equation, obtained by using terms of the ZPF field, was also proposed by HRP [18] whose form is

$$
H_{A}^{\prime}=\left(e^{2} \hbar / 2 \pi m_{0} c^{3}\right) \omega_{c}^{2}
$$

where $\hbar$ is a Plank constant divided by $2 \pi$ and $\omega_{c}$ is a suitable cutoff frequency of ZPF spectrum in the vacuum. Assuming that electrodynamics Hamiltonians, shown in (28) and (29), are identical with each other, we have $\Delta H_{A}=\Delta H_{A}^{\prime}$ for the dielectric material under an impressed electric field.
As suggested by Caligiuri [8], we suppose that the cutoff frequency of the vacuum is shifted as $\omega_{c}=\omega_{\text {Comp }}+\Delta \omega$ when the electromagnetic field is impressed to the dielectric material, which is similar to the assumption proposed by Musha by studying ZPF propulsion [19], consequently $\Delta H_{A}^{\prime}$ becomes

$$
\begin{aligned}
& \Delta H_{A}^{\prime}=\left(e^{2} \hbar / 2 \pi m_{0} c^{3}\right)\left\{\left(\omega_{\text {Comp }}+\Delta \omega\right)^{2}-\omega_{\text {Comp }}^{2}\right\} \\
& \approx\left(e^{2} \hbar / \pi m_{0} c^{3}\right) \omega_{\text {Comp }} \Delta \omega
\end{aligned}
$$

It is now important to note that the above equation can be expressed as a function of QV energy density $\rho_{Q V}$. This can be shown by generalizing (20) to a generic frequency value $\omega$

$$
\omega\left(\rho_{Q V}\right)=\left(c^{5} t_{P}^{2} \rho_{Q V} / \hbar\right)^{1 / 2}
$$

so that the shift of frequency, corresponding to a variation of QV energy density $\Delta \rho_{Q V}$, can be written as

$$
\Delta \omega=\omega^{\prime}-\omega=\omega\left(\rho_{Q V}+\Delta \rho_{Q^{v}}\right)-\omega\left(\rho_{Q V}\right)
$$

Supposing $\Delta \rho_{Q V} \rightarrow 0$ we can use a series development for $\omega\left(\rho_{Q_{v}}+\Delta \rho_{Q V}\right)$ at the first order in $\Delta \rho_{Q v}$, obtaining, after some simple mathematical manipulations

$$
\omega\left(\rho_{Q V}+\Delta \rho_{Q V}\right)=\left(c^{5} t_{P}^{2} \rho_{0} / \hbar\right)^{1 / 2}\left(1+\Delta \rho / 2 \rho_{0}\right)
$$

where $\rho_{0}$ is the QV energy density before the application of electric field. In particular, if we assume, as above, the resonance frequency to be equal to $\omega_{\text {Comp }}$ we have, by (31)

$$
\omega\left(\rho_{0}\right)=\omega_{\text {Comp }}
$$

and we can obtain, by solving (34)

$$
\rho_{0}=\hbar \omega_{\text {Comp }}^{2} / c^{5} t_{P}^{2}
$$

By using (33) into (32) we have

$$
\Delta \omega=\left(c^{5} t_{P}^{2} / \hbar \rho_{0}\right)^{1 / 2}(\Delta \rho / 2)
$$

so proving that the frequency shift $\Delta \omega$ is a function of $\mathrm{QV}$ energy density variation.

We can suppose that $H_{A}=0$ at the initial state, then we obtain the expression

$$
\Delta \omega \approx\left(\pi c / 2 \hbar \omega_{\text {Comp }}\right)<A^{2}>
$$

that can be written, by using the (35) and (36) into (37)

$$
\Delta \rho_{Q V} \approx\left(\pi / c^{4} t_{P}^{2}\right)<A^{2}>
$$

that gives the QV energy density variation corresponding to the application of a electromagnetic vector potential $\vec{A}$ to the vacuum for the considered apparatus. Equation (38) also generally proves that the application of an electromagnetic 
field to QV is able to modify, as supposed, its energy density.

\section{Force Generated in an Asymmetric Capacitor by an Applied Electric Field}

In a previous paper [20] Musha considered, for the capacitor composed of parallel plates, the possibility of the force generated by an external electric field from the standpoint of ZPF energy [20]. In spite of a parallel capacitor, let's consider an asymmetrical capacitor as shown in Fig. 3.

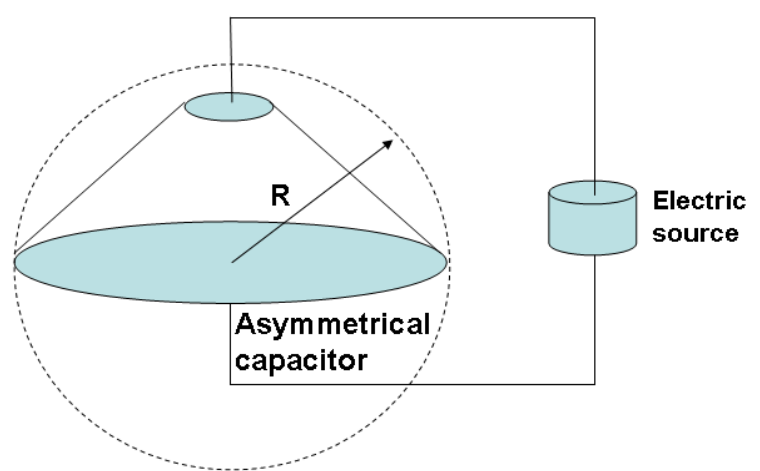

Figure 3. Asymmetrical capacitor under electric field ( $R$ indicates the size of electron cloud produced by electric field).

According to (37), the frequency of the ZPF field in a space around the capacitor under electric field can be represented as shown in Fig. 5.
(1)

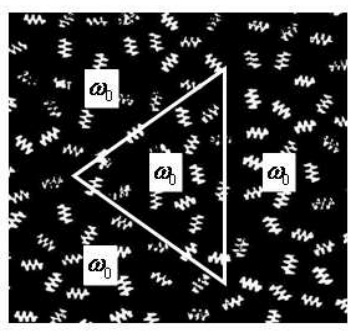

(2)

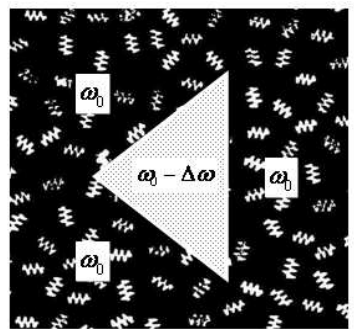

Figure 4. shows the frequency shift inside the capacitor before (1) and after (2) the electric field is applied to the triangle shaped asymmetrical capacitor.

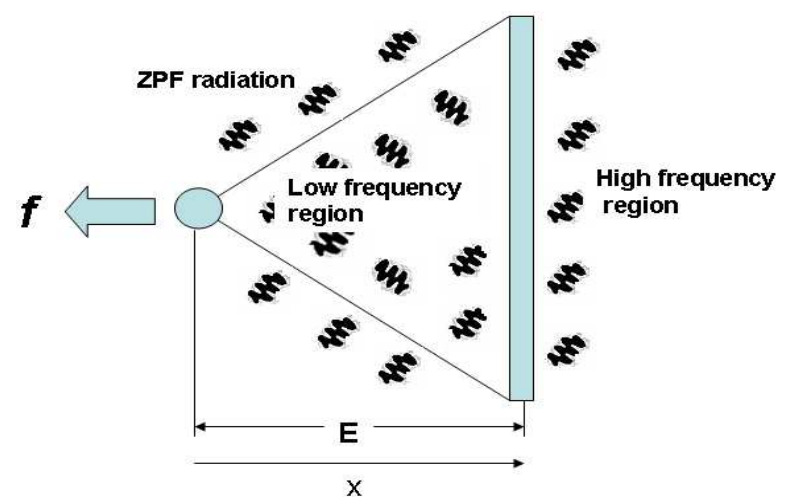

Figure 5. Frequencies of ZPF field around the shape of the capacitor.

The difference of energy along the $\mathrm{x}$-axis inside the capacitor before and after the electric field is applied to the capacitor is given by

$$
E^{\prime}=E_{1}-E_{2}=(\hbar \pi / 6)\left(R^{2} x^{3} / d^{2}\right) \Delta \omega
$$

where $R$ is the measure of the size of electron cloud (see Fig. 6) produced by the applied electric filed and $d$ is the distance between the capacitor plates.

From (37), the force generated at the end plate of the capacitor becomes

$$
\begin{aligned}
& \left.f \approx\left(\partial E^{\prime} / \partial x\right)\right|_{X=d}=\pi \hbar R^{2} \Delta \omega / 2= \\
& =\left(\pi^{2} c / 4\right)\left(R^{2} / \omega_{\text {Comp }}\right)<A^{2}>
\end{aligned}
$$

Using the results given by (38) and (27) we can write the above force as

$$
f=\left(\pi R^{2} c^{3} \hbar t_{P}^{2} / 4 m_{e}\right) \Delta \rho_{Q V}
$$

where $m_{e}$ is the electron rest mass.

From (41) we see that the force generated by the interaction between the electric field $\vec{E}$ and QV is directly proportional to QV energy density variation like in the Casimir effect.

Let's now consider the vector potential field generated by the variance of electric charges in an electron cloud shown in Fig. 6.

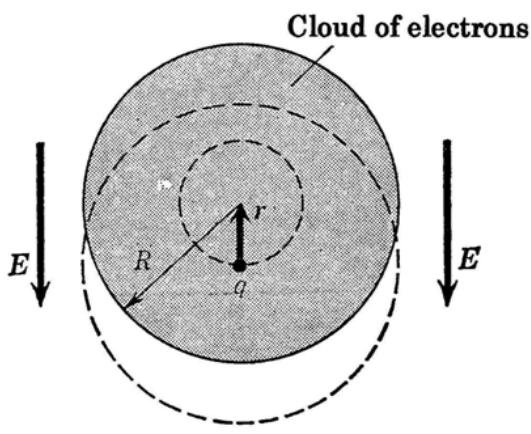

Figure 6. Vector potential field generated by an oscillating electric field.

The vector potential by an alternate electric field can be given by [21]

$$
\begin{aligned}
& A=\left(1 / 4 \pi \varepsilon_{0} c^{2}\right)\left(\omega p_{0} \cos \omega t / r\right)= \\
& =\left(1 / 4 \pi \varepsilon_{0} c^{2}\right)(\omega N e d \cos \omega t / r)
\end{aligned}
$$

where $N$ is a number density of electric charge and $d=(e / m)\left[E_{0} /\left(\omega_{e}^{2}-\omega^{2}\right)\right]$.

From (42), we have

$$
\begin{aligned}
& <A^{2}>=\left(N^{2} / 16 \pi^{2} \varepsilon_{0}^{2} c^{4}\right)\left(e^{4} / m^{2}\right) \times \\
& \left(\omega_{e}^{2} / \omega-\omega\right)^{-2}\left(E_{0}^{2} / 2 r^{2}\right)
\end{aligned}
$$

where $\omega_{e}$ is the resonant frequency of electrons given by $\omega_{e}=\sqrt{Z e^{2} / \alpha_{e} m}$ (where $\alpha_{e}$ is the electron polarizability). 
By integrating (43) in the volume of the electron cloud with the radius $R$, we have

$$
\begin{aligned}
& \int<A^{2}>d V=\int<A^{2}>\sin \theta d r d \theta d \phi= \\
& =\left(N^{2} e^{4} R / 8 \pi \varepsilon_{0}^{2} c^{4} m^{2}\right) X(\omega) E_{0}^{2}
\end{aligned}
$$

where $X(\omega)=\left(\omega_{e}^{2} / \omega-\omega\right)^{-2}$.

The value of $X(\omega)$ can be plotted as shown in Fig. 7, where we let $\omega_{e} \approx 10^{15} \mathrm{~Hz}$ ).

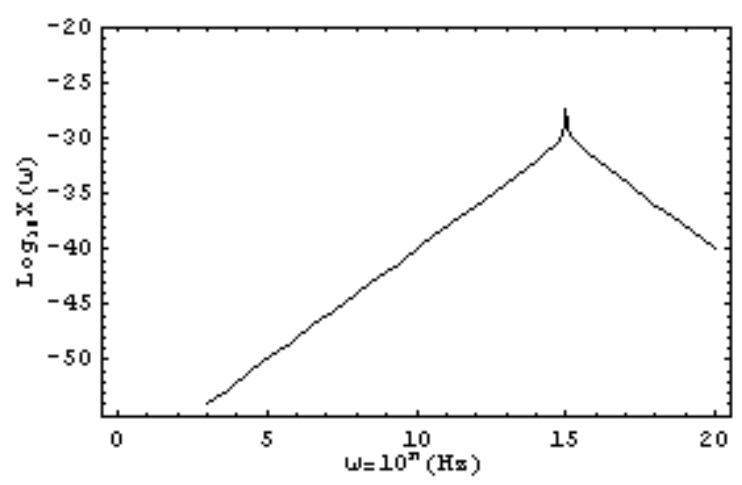

Figure 7. Plot of the function $X(\omega)$.

Hence we have

$$
\begin{aligned}
& f \approx\left(\pi^{2} c R / 4 \omega_{\text {Comp }}\right) \int<A^{2}>d V= \\
& =\left(N^{2} e^{4} R^{3} \pi / 32 \varepsilon_{0}^{2} c^{3} m^{2} \omega_{\text {Comp }}\right) X(\omega) E_{0}^{2}
\end{aligned}
$$

Thus from (45) it can be seen that a force can be generated at the end of the capacitor by applied electric field.

This equation suggests that an high potential impulsive electric field, which has wide frequency spectrum, impressed to the asymmetric capacitor can produce a rapid acceleration, which could be utilized for the space propulsion of a space ship. We also note the force given by (45) depends on QV energy density variation, via the vector potential associated to variable electric field $\vec{E}$ as shown in the above discussion. Furthermore, it is remarkable to observe that, from a dynamical point of view, the generated force $f$ is equivalent to an anti-gravitational pushing force.

This can be viewed by noting that, due to the positiveness of $<A^{2}>$, we also have $\Delta \rho_{Q V}>0$ by (38) and then a reduction of gravitational force due both to a decrease of $G$, through (14), and of inertial masses of the interacting particles according to (6).

\section{Conclusions}

In this paper it has been discussed the possibility to alter the inertia of a body by modifying the energy density of electromagnetic quantum vacuum $(\mathrm{QV})$, often called Zero Point Field (ZPF). By considering a model of QV based on energy density dynamics recently developed it has been shown the inertia of a body (considered as its inertial and gravitational mass content) and gravity strengths itself (described by the factor $G$ in Newton's law of gravitation) both can be considered as quantum variables, since they depend upon QV energy density.

According to this picture inertia and gravity can be then, in principle, manipulated by modifying the local QV energy density, by means of the electromagnetic interaction. These results are in very good agreement with those obtained some years ago by Hairsch, Rueda and Puthoff (HRP) in which inertial and gravitational masses as well as gravity were interpreted as the result of an electromagnetic reaction force of ZPF, the so called "quantum vacuum inertia hypothesis", of which the model proposed in this paper suggests a different understanding within a more general picture of reality.

It has been also shown, in particular, that an electromagnetic field could be able to shift the "cutoff" frequency predicted by HRP model for a given particle and, consequently, its inertial and gravitational mass, proving that the same effect is explainable, within the proposed QV energy density model, as a local variation of this energy density. This reveals a direct and deep relationship between electromagnetic fields and QV energy density variation that also seems to rules the gravitational strength quantified by the value of $G$.

Finally, we have discussed the application of the discussed theoretical model to the realization of an eventual space propulsion system by considering the interactions between the zero-point field of the QV and a high potential variable electric field generated within an asymmetrical capacitor. We have shown that the generated electromagnetic field is able to determine on the capacitor plates, as the result of field interaction with quantum vacuum ZPF fluctuations, a force manifesting as a sort of antigravity, directly related to QV energy density, whose variation is able to modify both the inertial mass and gravitational strength described by Newton's constant $G$. In this sense our results suggest that a pulsed electric fields applied to the spacecraft could produce artificial gravity sufficient for practical application to the space propulsion technology.

More generally the discussed results, although still preliminary, appear very interesting also from a theoretical point of view since they are able to give valuable and novel insights in the understanding of the relationship between gravity, electromagnetism and quantum vacuum dynamics as will be further pointed out in forthcoming and in progress publications.

\section{Notes}

This paper is based on the collaboration research on ZPF physics and space propulsion systems conducted by Prof. L. M. Caligiuri and Dr. T. Musha as scientific activities of Foundation of Physics Research Center (FoPRC) in Italy.

\section{References}

[1] H. G. B. Casimir, "On the attraction between two perfectly conducting plates", Proc. Kon. Ned. Akad. Van Weten., Vol. 51, No. 7, pp. 793 - 796 (1948). 
[2] S. K. Lamourex, "Demonstration of the Casimir force in the 0.6 to $6 \mathrm{~mm}$ range", Phys. Rev. Lett. Vol. 78, No. 1, pp. 793 - 796 (1948).

[3] P. W. Milonni, R. J. Cook, M. E. Goggin, "Radiation pressure from the vacuum: Physical interpretation of the Casimir force", Phys. Rev. A, Vol. 49, No. 2, pp. 678 - 694 (1994).

[4] A. D. Sakharov, "Vacuum Quantum Fluctuations in Curved Space and the Theory of Gravitation”, General Relativity and Gravitation, Vol. 32, No. 2, pp. 365 - 367 (2000).

[5] H. E. Puthoff, "Gravity as a zero-point-fluctuation force", Phys Rev. A, Vol.39, No.5, pp. 2333 - 2342 (1989).

[6] A. Rueda, B. Haisch, "Gravity and the Quantum Vacuum Inertia Hypothesis", arXiv:gr-qc/0504061v3 (2005).

[7] H. E. Puthoff, "Engineering the Zero - Point Field and Polarizable Vacuum for Interstellar Flight”, JBIS, Vol. 55, pp. 137 - 144 (2002).

[8] L.M. Caligiuri, "Quantum Vacuum Energy Density Dynamics and its consequences on Inertia and Gravitation", submitted for publication.

[9] L. M. Caligiuri, "The Emergence of Space - Time and Matter: Entropic or Geometro - Hydrondynamic Process ? A Comparison and Critical Review", Quantum Matter, Vol. 3, No. 3, pp. $249-255 \quad$ (2014). DOI: http:/dx.doi.org/10.1166/qm.2014.1120..

[10] L. M. Caligiuri, A. Sorli, "Relativistic Energy and Mass Originate from Homogeneity of Space and Time and from Quantum Vacuum Energy Density", American Journal of Modern Physics, Vol. 3, No. 2, pp. 51 - 59 (2014). DOI: 10.11648/j.ajmp.20140302.14.

[11] L. M. Caligiuri, A. Sorli, "Gravity Originates from Variable Energy Density of Quantum Vacuum", American Journal of Modern Physics, Vol. 3, No. 3, pp. 118 - 128 (2014). DOI: 10.11648/j.ajmp.20140303.11.
[12] C. Calvet, "The Quantum Vacuum Lepton / Photon Ratio", Journal of Theoretics, Vol. 4 No. 2, 2002.

[13] E.Podkletnov, R.Nieminen, "A possible gravitational force shielding by bulk YBa2Cu3O7-x superconductor," Physica C, Vol. 203, No. 49 pp.441-444 (2008).

[14] N. Li, D. Noever, T. Robertson, R. Koczor, W. Brantley, "Static Test for A Gravitational Force Coupled to Type II YBCO Superconductors", Physica C, Vol. 281, pp. 260 - 267 (1997).

[15] G. Modanese, "On the theoretical interpretation of E. Podklenotnov's experiment", I.N.F.N. - Trento, Extract from report UTF - 391/96, LANL gr-qc/9612022, presented at World Congress of the International Astronautical Federation, 1997, No. IAA-97-4.1.07.

[16] B. Haish, A. Rueda, H. E. Puthoff, "Physics of the zero-point field implication for inertia, gravitation and mass", Speculation in Science and Technology, 20 (1997) pp.99-114.

[17] H. E. Puthoff, "Can the vacuum be engineered for spaceflight applications? Overview of theory and experiments", Journal of Scientific Exploration, Vol.12, No.1, pp. 295-302(1998).

[18] B. Haish, R. Rueda, H. E. Puthoff, "Inertia as a zero-point-field Lorenz force”, Physical Review. A, Vol.49, No.2, pp. 678-694 (1994).

[19] T. Musha, "Possibility of the Space Propulsion System Utilizing the ZPF Field", Propulsion \& Energy Sciences International Forum-SPESIF-2009, American Institute of Physics (2009), pp.194-201.

[20] T.Musha, "Explanation of Dynamical Biefeld-Brown Effect From the Standpoint of ZPF Field", JBIS, Vol.61 (2008), pp.379-384.

[21] R.P.Feynmann, R.B.Leighton and M.Sands, The Feynman Lectures on Physics, Vol.II, Addison-Wesley,New York(1964). 